\title{
Indeterminate Forms
}

\author{
Dagnachew Jenber \\ University of Addis Ababa Science and Technology, Addis Ababa, Ethiopia \\ Email: djdm_101979@yahoo.com
}

Received 4 April 2016; accepted 22 July 2016; published 25 July 2016

Copyright () 2016 by author and Scientific Research Publishing Inc.

This work is licensed under the Creative Commons Attribution International License (CC BY).

http://creativecommons.org/licenses/by/4.0/

(c) () Open Access

\section{Abstract}

In this paper, $I^{\prime} m$ going to discuss on why are $\frac{0}{0}, \frac{ \pm \infty}{ \pm \infty},( \pm \infty)-( \pm \infty), 0 \cdot( \pm \infty), 0^{0},( \pm \infty)^{0}$, and $1^{( \pm \infty)}$ indeterminate form using simple calculus and algebra.

\section{Keywords}

Integration, Differentiation, Simple Algebra

\section{Introduction}

There are so many things that they are undetermined/indeterminate. Our universe is too complex to model mathematically because most of them are undetermined/indeterminate, but we have mathematical model for indeterminate things itself called indeterminate forms. For example, the weight and height of human beings is indeterminate because we can't say the weight or height of human being is this much.

Everything we don't know is infinitely many times more than everything we know. From something we know we can creat something we don't know and that gonna be something we know and using this something we know we can creat another something we don't know and it will continue and never ending. For example, let the only thing we know is hydrogen and oxgen but if we combine two hydrogen and one oxgen we can creat another thing, say water and by combining alot of water together we can creat another thing, say river, lake, ocean etc and also if we add something in to the river or lake or ocean we may creat another living things that we haven't seen them before and we can continue in such away that creating something we don't know from something we know.

Someone may ask the main question, "What is the thing before something ?". In other words, where is our universe comes from? Is our universe comes from something or from nothing? If we assume that our universe is created by God and then some one may ask, "Where God comes from and where he was before he creat the universe?" and Some one may answer for this question like "Before God there is nothing and God comes from nothing". This may indicates that there is something from nothing [1]-[13]. If there is something from nothing and we know that after something we will have an endless thing, so what is the common thing from nothing and 
an endless thing? according to our hypothesis it should be something. Therefore if we denote nothing by 0 and something by $c$, then $\frac{0}{0}=c$ since there is something from nothing and if there is something and using algebra, $\frac{0}{0}=c$ implies $0 \cdot( \pm \infty)=c$, where $\pm \infty$ is the notation for an endless thing/idea and in mathematics language which means doesn't exist. Here we can't say anything about nothing while nothing exists but invisible. Till now the first and the only thing we imagine about $c$ is, $c$ might be God or Big-bang. To be something exist there should be nothing exist first. Empty-space doesn't imply that nothing and vice versa since nothing is not measurable and not observable but existance [1]-[13]. In this paper, I am going to discuss on the proof of indeterminate things in our universe mathematically called indeterminate forms.

In calculus and other branches of mathematical analysis, limits involving algebraic combination of functions in an independent variable may often be evaluated by replacing these functions by their limits; if the expression obtained after this substitution does not give enough information to determine the original limit, it is said to take on an indeterminate form. The term was originally introduced by cauchy's student Moigno in the middle of the 19th century.

The indeterminate forms typically considered in the literature are denoted $\frac{0}{0}, \frac{\infty}{\infty}, 0(\infty), \infty-\infty, 0^{0}, 1^{\infty}$ and $\infty^{0}[14][15]$.

\section{Why Is $\frac{1}{0}$ Doesn't Exist?}

Definition: The function $\int_{0}^{\infty} f(t) \mathrm{e}^{-s t} \mathrm{~d} t$ is called the Laplace transform of the function $f(t)$. The variable $s$ is, in general, a complex variable.

Theorem 1. The number $\frac{a}{b}$ is doesn't exist for any positive real numbers $a \neq 0$ and $b=0$, that is, $\frac{a}{0}=\infty$. Proof. We know that the laplace transform of 1 is equal to $\frac{1}{s}$ for $s \neq 0$, that is,

$$
\int_{0}^{\infty} 1 \mathrm{e}^{-s t} \mathrm{~d} t=\frac{1}{s} \text { for any } s, t \in(0,+\infty] \text {. }
$$

But if $s=0$, the laplace transform of 1 becomes doesn't exist, that is,

$$
\int_{0}^{\infty} \mathrm{e}^{-0 t} \mathrm{~d} t=\frac{1}{0} \Rightarrow \lim _{k \rightarrow+\infty} \int_{0}^{k} \mathrm{~d} t=\frac{1}{0} \Rightarrow \lim _{k \rightarrow+\infty}(k)=\frac{1}{0} \Rightarrow \frac{1}{0}=+\infty .
$$

Therefore $\frac{1}{0}$ is doesn't exist and if we multiply this equation both sides by any non zero positive real numbers $a$, we get $\frac{a}{0}=+\infty$.

Corollary 1. $\frac{a}{0}=-\infty$ for $a<0$.

Proof. $a\left(\frac{1}{0}\right)=a(+\infty) \Rightarrow \frac{a}{0}=-\infty$ for any non zero negative real number $a$.

Theorem 2. The number $\frac{-a}{b}$ is doesn't exist for any positive real numbers $a \neq 0$ and $b=0$, that is, $\frac{-a}{0}=+\infty$.

Proof. We know that the laplace transform of 1 is equal to $\frac{1}{s}$ for $s \neq 0$, that is,

$$
\int_{0}^{\infty} 1 \mathrm{e}^{-s t} \mathrm{~d} t=\frac{1}{s} \text { for any } s, t \in(0,+\infty]
$$




$$
\begin{gathered}
\Rightarrow-\int_{0}^{-\infty} 1 \mathrm{e}^{s t} \mathrm{~d} t=\frac{1}{s} \text { for any } s \in(0,+\infty] \text { and } t \in[-\infty, 0) \quad \text { (integration by substitution.) } \\
\Rightarrow \int_{-\infty}^{0} 1 \mathrm{e}^{s t} \mathrm{~d} t=\frac{1}{s} \text { for any } s \in(0,+\infty] \text { and } t \in[-\infty, 0)
\end{gathered}
$$

Let's replace $s$ by $-s$, then we get

$$
\int_{-\infty}^{0} 1 \mathrm{e}^{-s t} \mathrm{~d} t=\frac{1}{-s}=\frac{-1}{s} \text { for any } s, t \in[-\infty, 0)
$$

But if $s=0$, the laplace transform of 1 becomes doesn't exist, that is,

$$
\int_{-\infty}^{0} 1 \mathrm{e}^{-0 t} \mathrm{~d} t=\frac{-1}{0} \Rightarrow \lim _{k \rightarrow-\infty} \int_{k}^{0} \mathrm{~d} t=\frac{-1}{0} \Rightarrow \lim _{k \rightarrow-\infty}(-k)=\frac{-1}{0} \Rightarrow \frac{-1}{0}=+\infty .
$$

Therefore $\frac{-1}{0}$ is doesn't exist and if we multiply this equation both sides by any non zero positive real numbers $a$, we get $\frac{-a}{0}=+\infty$.

Corollary 2. $\frac{-a}{0}=-\infty$ for $a<0$.

Proof. $a\left(\frac{-1}{0}\right)=a(+\infty)$ or $\frac{-a}{0}=-\infty$ for any non zero negative real numbers $a$.

Theorem 3. $\frac{1}{0}= \pm \infty$ and $\frac{-1}{0}= \pm \infty$.

Proof. Easily sketched from Theorem 1-2 and Corollary 1-2.

Theorem 4. The function $f(x)=\ln (x)$ is not defined for $x<0$ and doesn't exists for $x=0$, that is, $f(0)=\ln (0)=-\infty$.

Proof. From above Theorem-1 we have, $\frac{1}{0}=+\infty$

If we take natural logarithm both sides of this equation we get,

$$
\ln \left(\frac{1}{0}\right)=\ln (1)-\ln (0)=-\ln (0) \Rightarrow \ln (+\infty)=-\ln (0) \Rightarrow \ln (0)=-\infty
$$

\section{Why Is $\mathbf{0} \cdot( \pm \infty)$ Indeterminate?}

\section{Remark: For every natural numbers $\boldsymbol{m}$ and $\boldsymbol{n}$ such that $m \neq n$ we have,}

1. $0^{m}=0^{n}$.

2. $( \pm \infty)^{m}=( \pm \infty)^{n}$.

3. $1^{m}=1^{n}$.

4. $(( \pm \infty)-( \pm \infty))^{m}=(( \pm \infty)-( \pm \infty))^{n}$

Theorem 5. The number $a \cdot \frac{1}{b}$ is defined, exists but not unique for $a=b=0$, that is, $0 \cdot( \pm \infty)=c$, where $C \in \mathfrak{R}$.

Proof. Let's take any arbitrary constants $\alpha$ and $\beta$ such that $\alpha \neq \beta \neq 0$.

We know that $0^{m}=0^{n}$ then $(0)^{m}(\alpha)=(0)^{n}(\beta)$, if we divide the left and right hand side of this equation by $0^{m}$ and $0^{n}$ respectively, then we get

$$
\begin{aligned}
(0)^{m}\left(\frac{\alpha}{0^{m}}\right) & =(0)^{n}\left(\frac{\beta}{0^{n}}\right) \Rightarrow(0)^{m}\left(\frac{1^{m}}{0^{m}}\right)(\alpha)=(0)^{n}\left(\frac{1^{n}}{0^{n}}\right)(\beta) \\
\Rightarrow(0)^{m}\left(\frac{1}{0}\right)^{m}(\alpha) & =(0)^{n}\left(\frac{1}{0}\right)^{n}(\beta) \Rightarrow(0)^{m}( \pm \infty)^{m}(\alpha)=(0)^{n}( \pm \infty)^{n}(\beta)
\end{aligned}
$$


this implies that for every natural numbers $m$ and $n$

$$
\begin{gathered}
(0 \cdot \pm \infty)^{m}(\alpha)=(0 \cdot \pm \infty)^{n}(\beta) \Rightarrow(0 \cdot \pm \infty)^{m}(\alpha)-(0 \cdot \pm \infty)^{n}(\beta)=0 \\
\Rightarrow(0 \cdot \pm \infty)^{m}\left(\alpha-(0 \cdot \pm \infty)^{n-m}(\beta)\right)=0 \Rightarrow(0 \cdot \pm \infty)^{m}=0 \text { or }\left(\alpha-(0 \cdot \pm \infty)^{n-m}(\beta)\right)=0 \\
\Rightarrow(0 \cdot \pm \infty)=0^{\frac{1}{m}} \text { or }(0 \cdot \pm \infty)^{n-m}=\frac{\alpha}{\beta} \Rightarrow(0 \cdot \pm \infty)=0 \text { or }(0 \cdot \pm \infty)=\left(\frac{\alpha}{\beta}\right)^{\frac{1}{n-m}} \text { which is complex number. }
\end{gathered}
$$

From this we must therfore conclude that $(0 \cdot \pm \infty)$ is defined, exists but not unique so indeterminate.

Theorem 6. If $0(+\infty)=C \in \mathfrak{R}^{+}$and $0(-\infty)=c \in \mathfrak{R}^{-}$, then $0 \neq-0$.

Proof. Suppose $0=-0,0(+\infty)=C \in \mathfrak{R}^{+}$and $0(-\infty)=c \in \mathfrak{R}^{-}$, then

$$
C=(0)(+\infty)=(-0)(+\infty)=(0)(-1)(+\infty)=(0)(-\infty)=c \Rightarrow c=C \text { (contradiction) }
$$

Therefore $0 \neq-0$.

This theorem shows that $(0)(+\infty)=\alpha \in \mathfrak{R}$ and $(0)(-\infty)=\beta \in \mathfrak{R}$ or $(0)( \pm \infty)=\gamma \in \mathfrak{R}$ since we know that $0=-0$.

Theorem 7. The number $a \cdot \frac{1}{b}$ is defined, exists but not unique for $a=b=0$, that is, $0 \cdot( \pm \infty)=c$, where $c \in \Re$.

Proof. Suppose $x \neq 0$ is any given real number, then

$$
\begin{aligned}
& \frac{\mathrm{d}}{\mathrm{d} x}\left((x)\left(\frac{1}{x}\right)\right)=(x) \frac{\mathrm{d}}{\mathrm{d} x}\left(\frac{1}{x}\right)+\left(\frac{1}{x}\right) \frac{\mathrm{d}}{\mathrm{d} x}(x) \Rightarrow \mathrm{d}\left((x)\left(\frac{1}{x}\right)\right)=\left((x) \frac{\mathrm{d}}{\mathrm{d} x}\left(\frac{1}{x}\right)+\left(\frac{1}{x}\right) \frac{\mathrm{d}}{\mathrm{d} x}(x)\right) \mathrm{d} x \\
\Rightarrow & \int \mathrm{d}\left((x)\left(\frac{1}{x}\right)\right)=\int\left((x) \frac{\mathrm{d}}{\mathrm{d} x}\left(\frac{1}{x}\right)+\left(\frac{1}{x}\right) \frac{\mathrm{d}}{\mathrm{d} x}(x)\right) \mathrm{d} x \Rightarrow(x)\left(\frac{1}{x}\right)=\int\left((x) \frac{\mathrm{d}}{\mathrm{d} x}\left(\frac{1}{x}\right)+\left(\frac{1}{x}\right) \frac{\mathrm{d}}{\mathrm{d} x}(x)\right) \mathrm{d} x
\end{aligned}
$$

$\Rightarrow 1=c$ for $x \neq 0$ from our assumption, where $c \in \mathfrak{R}$ is the integration of 0.

Therefore $x\left(\frac{1}{x}\right)=c=1$ or $x\left(\frac{1}{x}\right)=1$ for $x \neq 0$ is defined, exists and unique.

Let's suppose that $x=0$ then our equation becomes $0\left(\frac{1}{0}\right)=0 \cdot( \pm \infty)=c$ where $c$ is the integration of 0 which is any real number and this indicates that $0 \cdot( \pm \infty)$ is defined, exists but not unique.

Thus $0 \cdot( \pm \infty)$ is indeterminate form.

\section{Why Is $\frac{0}{0}$ Indeterminate?}

Theorem 8. The number $\frac{a}{b}$ is defined, exists but not unique for $a=0$ and $b=0$, that is, $\frac{0}{0}=c$ where $c \in \mathfrak{R}$.

Proof. Let's take any arbitrary constants $\alpha$ and $\beta$ such that $\alpha \neq \beta \neq 0$.

We know that $0^{m}=0^{n}$ then $(0)^{m}(\alpha)=(0)^{n}(\beta)$, if we divide the left and right hand side of this equation by $0^{m}$ and $0^{n}$ respectively, then we get

$$
\left(\frac{0^{m}}{0^{m}}\right) \alpha=\left(\frac{0^{n}}{0^{n}}\right) \beta \Rightarrow\left(\frac{0}{0}\right)^{m} \alpha=\left(\frac{0}{0}\right)^{n} \beta
$$

this implies that for every natural numbers $m$ and $n$,

$$
\left(\frac{0}{0}\right)^{m}(\alpha)-\left(\frac{0}{0}\right)^{n}(\beta)=0 \Rightarrow\left(\frac{0}{0}\right)^{m}\left(\alpha-\left(\frac{0}{0}\right)^{n-m}(\beta)\right)=0
$$




$$
\Rightarrow\left(\frac{0}{0}\right)^{m}=0 \text { or }\left(\alpha-\left(\frac{0}{0}\right)^{n-m}(\beta)\right)=0 \Rightarrow\left(\frac{0}{0}\right)=0 \text { or }\left(\frac{0}{0}\right)=\left(\frac{\alpha}{\beta}\right)^{\frac{1}{n-m}} \text { which is complex number }
$$

From this we must therefore conclude that $\frac{0}{0}$ is defined, exists but not unique so indeterminate.

Theorem 9. The number $\frac{a}{b}$ is defined, exists but not unique for $a=0$ and $b=0$, that is, $\frac{0}{0}=c$ where $c \in \Re$.

Proof. Since $\frac{1}{0}= \pm \infty$, then $\frac{0}{0}=0\left(\frac{1}{0}\right)=0( \pm \infty)=c$ this indicates that the number $\frac{0}{0}$ is defined, exists but not unique since $c$ is any arbitrarary constant so indeterminate form.

Theorem 10. The number $\frac{a}{b}$ is defined, exists but not unique for $a=0$ and $b=0$, that is, $\frac{0}{0}=c$ where $c \in \mathfrak{R}$.

Proof. Suppose $x \neq 0$ is any given real number, then

$$
\begin{aligned}
\frac{\mathrm{d}}{\mathrm{d} x}\left(\frac{x}{x}\right) & =\frac{(x) \frac{\mathrm{d}}{\mathrm{d} x}(x)-(x) \frac{\mathrm{d}}{\mathrm{d} x}(x)}{x^{2}} \Rightarrow \mathrm{d}\left(\frac{x}{x}\right)=\left(\frac{(x) \frac{\mathrm{d}}{\mathrm{d} x}(x)-(x) \frac{\mathrm{d}}{\mathrm{d} x}(x)}{x^{2}}\right) \mathrm{d} x \\
\Rightarrow \int \mathrm{d}\left(\frac{x}{x}\right) & =\int\left(\frac{(x) \frac{\mathrm{d}}{\mathrm{d} x}(x)-(x) \frac{\mathrm{d}}{\mathrm{d} x}(x)}{x^{2}}\right) \mathrm{d} x \Rightarrow \frac{x}{x}=\int\left(\frac{(x) \frac{\mathrm{d}}{\mathrm{d} x}(x)-(x) \frac{\mathrm{d}}{\mathrm{d} x}(x)}{x^{2}}\right) \mathrm{d} x
\end{aligned}
$$

but from our assumption $x \neq 0$, then we have $1=\int 0 \mathrm{~d} x=c$.

Therefore $\frac{x}{x}=c=1$ or $\frac{x}{x}=1$ for $x \neq 0$ is defined, exists and unique.

Let's suppose that $x=0$, then our equation becomes $\frac{0}{0}=c$ where $c$ is the integration of 0 which is any real number and this indicates that $\frac{0}{0}$ is defined, exists but not unique.

Thus $\frac{0}{0}$ is indeterminate form.

\section{Why Is $\frac{ \pm \infty}{ \pm \infty}$ Indeterminate?}

Theorem 11. The number $\frac{\frac{1}{a}}{\frac{1}{b}}$ is defined, exists but not unique for $a=0$ and $b=0$, that is, $\frac{ \pm \infty}{ \pm \infty}=c$ where $c \in \Re$.

Proof. Let's take any arbitrary constants $\alpha$ and $\beta$ such that $\alpha \neq \beta \neq 0$.

We know that $( \pm \infty)^{m}=( \pm \infty)^{n}$ then $( \pm \infty)^{m}(\alpha)=( \pm \infty)^{n}(\beta)$, if we divide the left and right hand side of this equation by $( \pm \infty)^{m}$ and $( \pm \infty)^{n}$ respectively, then we get

$$
\left(\frac{( \pm \infty)^{m}}{( \pm \infty)^{m}}\right) \alpha=\left(\frac{( \pm \infty)^{n}}{( \pm \infty)^{n}}\right) \beta \Rightarrow\left(\frac{ \pm \infty}{ \pm \infty}\right)^{m} \alpha=\left(\frac{ \pm \infty}{ \pm \infty}\right)^{n} \beta
$$

this implies that for every natural numbers $m$ and $n$ such that $m \neq n$, 


$$
\begin{gathered}
\left(\frac{ \pm \infty}{ \pm \infty}\right)^{m}(\alpha)-\left(\frac{ \pm \infty}{ \pm \infty}\right)^{n}(\beta)=0 \Rightarrow\left(\frac{ \pm \infty}{ \pm \infty}\right)^{m}\left(\alpha-\left(\frac{ \pm \infty}{ \pm \infty}\right)^{n-m}(\beta)\right)=0 \\
\Rightarrow\left(\frac{ \pm \infty}{ \pm \infty}\right)^{m}=0 \text { or }\left(\alpha-\left(\frac{ \pm \infty}{ \pm \infty}\right)^{n-m}(\beta)\right)=0 \Rightarrow\left(\frac{ \pm \infty}{ \pm \infty}\right)=0 \text { or }\left(\frac{ \pm \infty}{ \pm \infty}\right)=\left(\frac{\alpha}{\beta}\right)^{\frac{1}{n-m}} \text { which is complex number }
\end{gathered}
$$

From this we must therefore conclude that $\frac{ \pm \infty}{ \pm \infty}$ is defined, exists but not unique so indeterminate.

Theorem 12. The number $\frac{\frac{1}{a}}{\frac{1}{b}}$ is defined, exists but not unique for $a=0$ and $b=0$, that is, $\frac{ \pm \infty}{ \pm \infty}=c$ where $c \in \mathfrak{R}$.

Proof. Suppose $x \neq 0$ is any given real number, then

$$
\begin{aligned}
& \frac{\mathrm{d}}{\mathrm{d} x}\left(\frac{\left(\frac{1}{x}\right)}{\left(\frac{1}{x}\right)}\right)=\frac{\left(\frac{1}{x}\right) \frac{\mathrm{d}}{\mathrm{d} x}\left(\frac{1}{x}\right)-\left(\frac{1}{x}\right) \frac{\mathrm{d}}{\mathrm{d} x}\left(\frac{1}{x}\right)}{\left(\frac{1}{x}\right)^{2}} \Rightarrow \mathrm{d}\left(\frac{\left(\frac{1}{x}\right)}{\left(\frac{1}{x}\right)}\right)=\left(\frac{\left(\frac{1}{x}\right) \frac{\mathrm{d}}{\mathrm{d} x}\left(\frac{1}{x}\right)-\left(\frac{1}{x}\right) \frac{\mathrm{d}}{\mathrm{d} x}\left(\frac{1}{x}\right)}{\left(\frac{1}{x}\right)^{2}}\right) \mathrm{d} x \\
\Rightarrow \int \mathrm{d}\left(\frac{\left(\frac{1}{x}\right)}{\left(\frac{1}{x}\right)}\right) & =\int\left(\frac{\left(\frac{1}{x}\right) \frac{\mathrm{d}}{\mathrm{d} x}\left(\frac{1}{x}\right)-\left(\frac{1}{x}\right) \frac{\mathrm{d}}{\mathrm{d} x}\left(\frac{1}{x}\right)}{\left(\frac{1}{x}\right)^{2}}\right) \mathrm{d} x \Rightarrow \frac{\left(\frac{1}{x}\right)}{\left(\frac{1}{x}\right)}=\int\left(\frac{\left(\frac{1}{x}\right) \frac{\mathrm{d}}{\mathrm{d} x}\left(\frac{1}{x}\right)-\left(\frac{1}{x}\right) \frac{\mathrm{d}}{\mathrm{d} x}\left(\frac{1}{x}\right)}{\left(\frac{1}{x}\right)^{2}}\right) \mathrm{d} x
\end{aligned}
$$

but from our assumption $x \neq 0$, then we have $1=\int 0 \mathrm{~d} x=c$.

Therefore $\frac{\left(\frac{1}{x}\right)}{\left(\frac{1}{x}\right)}=c=1$ or $\frac{\left(\frac{1}{x}\right)}{\left(\frac{1}{x}\right)}=1$ for $x \neq 0$ is defined, exists and unique.

Let's suppose that $x=0$, then our equation becomes $\frac{\left(\frac{1}{0}\right)}{\left(\frac{1}{0}\right)}=\frac{ \pm \infty}{ \pm \infty}=c$ where $c$ is the integration of 0 which is any real number and this indicates that $\frac{ \pm \infty}{ \pm \infty}$ is defined, exists but not unique.

Thus $\frac{ \pm \infty}{ \pm \infty}$ is indeterminate form.

\section{Why Is $( \pm \infty)-( \pm \infty)$ Indeterminate?}

Theorem 13. The number $\frac{1}{a}-\frac{1}{b}$ is defined, exists but not unique for $a=b=0$, that is, $( \pm \infty)-( \pm \infty)=c$, where $c \in \mathfrak{R}$.

Proof. Let's take any arbitrary constants $\alpha$ and $\beta$ such that $\alpha \neq \beta \neq 0$.

We know that $(( \pm \infty)-( \pm \infty))^{m}=(( \pm \infty)-( \pm \infty))^{n}$ then $(( \pm \infty)-( \pm \infty))^{m}(\alpha)=(( \pm \infty)-( \pm \infty))^{n}(\beta)$.

Thus for every natural numbers $m$ and $n$ such that $m \neq n$, we have

$$
(( \pm \infty)-( \pm \infty))^{m}(\alpha)=(( \pm \infty)-( \pm \infty))^{n}(\beta) \Rightarrow(( \pm \infty)-( \pm \infty))^{m}(\alpha)-(( \pm \infty)-( \pm \infty))^{n}(\beta)=0
$$




$$
\begin{gathered}
\Rightarrow(( \pm \infty)-( \pm \infty))^{m}\left(\alpha-(( \pm \infty)-( \pm \infty))^{n-m}(\beta)\right)=0 \Rightarrow(( \pm \infty)-( \pm \infty))^{m}=0 \text { or }\left(\alpha-(( \pm \infty)-( \pm \infty))^{n-m}(\beta)\right)=0 \\
\Rightarrow(( \pm \infty)-( \pm \infty))=0 \text { or }(( \pm \infty)-( \pm \infty))=\left(\frac{\alpha}{\beta}\right)^{\frac{1}{n-m}} \text { which is complex. }
\end{gathered}
$$

From this we must therefore conclude that $( \pm \infty)-( \pm \infty)$ is defined, exists but not unique so indeterminate.

Theorem 14. The number $\frac{1}{a}-\frac{1}{b}$ is defined, exists but not unique for $a=b=0$, that is, $( \pm \infty)-( \pm \infty)=c$, where $c \in \Re$.

Proof. Suppose $x \neq 0$ is any given real number, then

$$
\begin{aligned}
& \frac{\mathrm{d}}{\mathrm{d} x}\left(\frac{1}{x}-\frac{1}{x}\right)=\frac{\mathrm{d}}{\mathrm{d} x}\left(\frac{1}{x}\right)-\frac{\mathrm{d}}{\mathrm{d} x}\left(\frac{1}{x}\right) \Rightarrow \mathrm{d}\left(\frac{1}{x}-\frac{1}{x}\right)=\left(\frac{\mathrm{d}}{\mathrm{d} x}\left(\frac{1}{x}\right)-\frac{\mathrm{d}}{\mathrm{d} x}\left(\frac{1}{x}\right)\right) \mathrm{d} x \\
\Rightarrow & \int \mathrm{d}\left(\frac{1}{x}-\frac{1}{x}\right)=\int\left(\frac{\mathrm{d}}{\mathrm{d} x}\left(\frac{1}{x}\right)-\frac{\mathrm{d}}{\mathrm{d} x}\left(\frac{1}{x}\right)\right) \mathrm{d} x \Rightarrow \frac{1}{x}-\frac{1}{x}=\int\left(\frac{\mathrm{d}}{\mathrm{d} x}\left(\frac{1}{x}\right)-\frac{\mathrm{d}}{\mathrm{d} x}\left(\frac{1}{x}\right)\right) \mathrm{d} x
\end{aligned}
$$

$\Rightarrow 0=c$ for $x \neq 0$ from our assumption and $c \in \mathfrak{R}$ is the integration of 0.

Therefore $\frac{1}{x}-\frac{1}{x}=c=0$ or $\frac{1}{x}-\frac{1}{x}=0$ for $x \neq 0$ is defined, exists and unique.

Let's suppose that $x=0$ then our equation becomes $\frac{1}{0}-\frac{1}{0}=( \pm \infty)-( \pm \infty)=c$ where $c$ is the integration of 0 which is any real number and this indicates that $( \pm \infty)-( \pm \infty)$ is defined, exists but not unique.

Thus $( \pm \infty)-( \pm \infty)$ is indeterminate form.

\section{Why Is $0^{0}$ Indeterminate?}

Theorem 15. The number $a^{b}$ is defined, exists but not unique for $a=b=0$, that is, $0^{0}=C$, where $C \in \mathfrak{R}^{+} \cup\{0,+\infty\}$.

Proof. We know that $x^{x}=\mathrm{e}^{\ln \left(x^{x}\right)}=\mathrm{e}^{x \ln (x)}$ for any positive real numbers $x$.

But if $x=0$, then $0^{0}=\mathrm{e}^{0 \ln (0)}=\mathrm{e}^{0(-\infty)}=\mathrm{e}^{c}=C \quad$ or $0^{0}=C \in \mathfrak{R}^{+} \cup\{0,+\infty\}$ for $c \in \mathfrak{R}$, where $C=0(c \rightarrow-\infty)$ and $C=+\infty(c \rightarrow+\infty)$ this indicates that the number $0^{0}$ is defined, exists but not unique so indeterminate form.

\section{Why Is $\mathbb{1}^{( \pm \infty)}$ Indeterminate?}

Theorem 16. The number $a^{\frac{1}{b}}$ is defined, exists but not unique for $a=1$ and $b=0$, that is, $1^{\infty}=C$, where $C \in \mathfrak{R}^{+} \cup\{0,+\infty\}$.

Proof. We know that $y^{\left(\frac{1}{x}\right)}=\mathrm{e}^{\ln \left(y^{\left(\frac{1}{x}\right)}\right)}=\mathrm{e}^{\frac{\ln (y)}{x}}$ for any real numbers $x$ and positive real numbers $y$.

But if $y=1$ and $x=0$, then $y^{\left(\frac{1}{x}\right)}=1^{\left(\frac{1}{0}\right)}=\mathrm{e}^{\frac{\ln (1)}{0}}=\mathrm{e}^{\left(\frac{0}{0}\right)}=\mathrm{e}^{c}=C$ or $1^{\left(\frac{1}{0}\right)}=1^{(+\infty)}=\mathrm{e}^{c}=C \in \mathfrak{R}^{+} \cup\{0,+\infty\}$ for $c \in \mathfrak{R}$, where $C=0(c \rightarrow-\infty)$ and $C=+\infty(c \rightarrow+\infty)$ this indicates that the number $1^{(+\infty)}$ is defined, exists but not unique so indeterminate form.

Corollary 3. $1^{(-\infty)}=C \in \mathfrak{R}^{+} \cup\{0,+\infty\}$.

Proof. We know that $y^{\left(-\frac{1}{x}\right)}=\mathrm{e}^{\ln \left(y^{\left(-\frac{1}{x}\right)}\right)}=\mathrm{e}^{\left((\ln (y))\left(-\frac{1}{x}\right)\right)}$ for any real numbers $x$ and positive real numbers $y$. 
But if $y=1$ and $x=0$, then $y^{\left(-\frac{1}{x}\right)}=1^{\left(-\frac{1}{0}\right)}=\mathrm{e}^{\left((\ln (1))\left(-\frac{1}{0}\right)\right)}=\mathrm{e}^{\left((0)\left(-\frac{1}{0}\right)\right)}=\mathrm{e}^{0 \cdot(-\infty)}=\mathrm{e}^{c}=C$ or $1^{\left(-\frac{1}{0}\right)}=1^{(-\infty)}=\mathrm{e}^{c}=C \in \mathfrak{R}^{+} \cup\{0,+\infty\}$ for $c \in \mathfrak{R}$, where $C=0(c \rightarrow-\infty)$ and $C=+\infty(c \rightarrow+\infty)$ this indicates that the number $1^{(-\infty)}$ is defined, exists but not unique so indeterminate form.

\section{Why Is $( \pm \infty)^{0}$ Indeterminate?}

Theorem 17. The number $\frac{1^{b}}{a}$ is defined, exists but not unique for $a=b=0$, that is, $\infty^{0}=C$, where $C \in \mathfrak{R}^{+} \cup\{0,+\infty\}$.

Proof. We know that $\left(\frac{1}{x}\right)^{x}=\mathrm{e}^{\ln \left(\left(\frac{1}{x}\right)^{x}\right)}=\mathrm{e}^{\left((x)\left(\ln \left(\frac{1}{x}\right)\right)\right)}$ for any positive real numbers $x$.

But if $x=0$, then

$$
\left(\frac{1}{x}\right)^{x}=\left(\frac{1}{0}\right)^{0}=\mathrm{e}^{\left((0)\left(\ln \left(\frac{1}{0}\right)\right)\right)}=\mathrm{e}^{((0)(\ln (+\infty)))}=\mathrm{e}^{(0)(+\infty)}=\mathrm{e}^{c}=C \text { or }\left(\frac{1}{0}\right)^{0}=(+\infty)^{0}=\mathrm{e}^{c}=C \in \mathfrak{R}^{+} \cup\{0,+\infty\}
$$

for $c \in \mathfrak{R}$, where $C=0(c \rightarrow-\infty)$ and $C=+\infty(c \rightarrow+\infty)$ this indicates that the number $(+\infty)^{0}$ is defined, exists but not unique so indeterminate form.

Corollary 4. $(-\infty)^{0}=C \in \mathfrak{R}^{+} \cup\{0,+\infty\}$.

Proof. We know that $\left(-\frac{1}{x}\right)^{x}=\mathrm{e}^{\ln \left(\left(-\frac{1}{x}\right)^{x}\right)}=\mathrm{e}^{\left((x)\left(\ln \left(-\frac{1}{x}\right)\right)\right)}=\mathrm{e}^{\left((x)\left(\ln \left(\frac{1}{(-x)}\right)\right)\right)}=\mathrm{e}^{((x)(\ln (1)-\ln (-x)))}=\mathrm{e}^{((x)(-\ln (-x)))}$ for any negative real numbers $x$.

But if $x=0$, then $\left(-\frac{1}{x}\right)^{x}=\left(-\frac{1}{0}\right)^{0}=\mathrm{e}^{((0)(-\ln (-0)))}=\mathrm{e}^{((0)(-\ln (0)))}=\mathrm{e}^{(0)(-(-\infty))}=\mathrm{e}^{(0)(+\infty)}=\mathrm{e}^{c}=C$ or $\left(-\frac{1}{0}\right)^{0}=(-\infty)^{0}=\mathrm{e}^{c}=C \in \mathfrak{R}^{+} \cup\{0,+\infty\}$ for $c \in \mathfrak{R}$, where $C=0(c \rightarrow-\infty)$ and $C=+\infty(c \rightarrow+\infty)$ this indicates that the number $(-\infty)^{0}$ is defined, exists but not unique so indeterminate form.

\section{References}

[1] Reynosa, P. (2016) Some of the Changes Lawrence M. Krauss Should Make to the Second Edition of "A Universe from Nothing”. Huffington Post.

[2] Krauss, L.M. (2012) A Universe from Nothing: Why There Is Something Rather than Nothing. Free Press, New York, 183.

[3] Andersen, R. (2014) Has Physics Made Philosophy and Religion Obsolete? http://www.theatlantic.com/

[4] Krauss, L. After Word from Lawrence Krauss’ New Book-A Universe from Nothing.

[5] Lawrence Krauss’s 2009 Lecture. A Universe from Nothing.

[6] “Non Fiction Best Sellers”. The New York Times.

[7] Albert, D. (2012) On the Origin of Everything. The New York Times Sunday Book Review.

[8] A Universe from Nothing?_Cosmic Variance: Cosmic Variance. http://blogs.discovermagazine.com/

[9] Physicist George Ellis Knocks Physicists for Knocking Philosophy, Falsification, Free Will/ Cross Check, Scientific American Blog Network. http://blogs.scientificamerican.com/

[10] Kohli, L.S. (2014) Comments on: A Universe from Nothing. arXiv.1405.6091 [physics.gen-ph].

[11] Scharf, C. (2012) Cosmology: Plucked from the Vacuum. Nature, 481, 440.

[12] Nelson, S. (2012) A Universe from Nothing. Review. The A.V. Club. 
[13] Brooks, M. (2012) The Paradox of Nothing. New Scientist, 213.

[14] Indeterminate Form-Wikipedia. https://en.m.wikipedia.org

[15] Rotando, L.M. and Korn, H. (1977) The Indeterminate Form $0^{0}$. Mathematics Magazine, 50, 41-42. http://dx.doi.org/10.2307/2689754

\section{Submit or recommend next manuscript to SCIRP and we will provide best service for you:}

Accepting pre-submission inquiries through Email, Facebook, LinkedIn, Twitter, etc.

A wide selection of journals (inclusive of 9 subjects, more than 200 journals)

Providing 24-hour high-quality service

User-friendly online submission system

Fair and swift peer-review system

Efficient typesetting and proofreading procedure

Display of the result of downloads and visits, as well as the number of cited articles

Maximum dissemination of your research work

Submit your manuscript at: http://papersubmission.scirp.org/ 